This postprint is published in:

The Journal of symbolic logic, Volume 80, Number 4, 2015, 1398-1410

DOI: http://dx.doi.org/10.1017/jsl.2014.73

\title{
MATHIAS FORCING AND COMBINATORIAL COVERING PROPERTIES OF FILTERS
}

\author{
D. CHODOUNSKÝ, D. REPOVŠ, AND L. ZDOMSKYY
}

\begin{abstract}
We give topological characterizations of filters $\mathcal{F}$ on $\omega$ such that the Mathias forcing $\mathbb{M}_{\mathcal{F}}$ adds no dominating reals or preserves ground model unbounded families. This allows us to answer some questions of Brendle, Guzmán, Hrušák, Martínez, Minami, and Tsaban.
\end{abstract}

\section{INTRODUCTION}

A subset $\mathcal{F}$ of $[\omega]^{\omega}$ is called a filter if $\mathcal{F}$ contains all co-finite sets, is closed under finite intersections of its elements, and under taking supersets. Every filter $\mathcal{F}$ gives rise to a natural forcing notion $\mathbb{M}_{\mathcal{F}}$ introducing a generic subset $X \in[\omega]^{\omega}$ such that $X \subset^{*} F$ for all $F \in \mathcal{F}$ as follows: $\mathbb{M}_{\mathcal{F}}$ consists of pairs $\langle s, F\rangle$ such that $s \in[\omega]<\omega, F \in \mathcal{F}$, and $\max s<\min F$. A condition $\langle s, F\rangle$ is stronger than $\langle t, G\rangle$ if $F \subset G, s$ is an end-extension of $t$, and $s \backslash t \subset G$. $\mathbb{M}_{\mathcal{F}}$ is usually called Mathias forcing associated with $\mathcal{F}$.

Posets of the form $\mathbb{M}_{\mathcal{F}}$ are important in the set theory of reals and have been used to establish various consistency results, see, e.g., [7, 12] and references therein. One of the most fundamental questions about $\mathbb{M}_{\mathcal{F}}$ is whether it adds a dominating real, i.e., whether in $\omega^{\omega}$ of the generic extension $V^{\mathbb{M}_{\mathcal{F}}}$ there exists $x$ such that for every $a \in \omega^{\omega}$ in the ground model $V$ the inequality $a(n) \leq x(n)$ holds for all but finitely many $n$. Such filters $\mathcal{F}$ admit the following topological characterization proved in section 2 .

Theorem 1.1. Let $\mathcal{F}$ be a filter. Then $\mathbb{M}_{\mathcal{F}}$ does not add dominating reals if and only if $\mathcal{F}$ has the Menger covering property as a subspace of $\mathcal{P}(\omega)$.

Recall from [15] that a topological space $X$ has the Menger covering property (or simply is Menger), if for every sequence $\left\langle\mathcal{U}_{n}: n \in \omega\right\rangle$ of open covers of $X$ there exists a sequence $\left\langle\mathcal{V}_{n}: n \in \omega\right\rangle$ such that $\mathcal{V}_{n} \in\left[\mathcal{U}_{n}\right]^{<\omega}$ and $\left\{\bigcup \mathcal{V}_{n}: n \in \omega\right\}$ is a cover of $X$. Menger spaces can be equivalently characterized as spaces $X$ such that no image of $X$ via a continuous function from $X$ to $\omega^{\omega}$ is $<^{*}$-dominating.

Theorem 1.1 has a number of applications. For instance, since analytic Menger sets of reals are $\sigma$-compact [1], it implies the following fact ${ }^{1}$ answering $[12$, Question 4.3] in the negative.

2010 Mathematics Subject Classification. Primary: 54D20, 03E40. Secondary: 54H05, $03 \mathrm{E} 05$.

Key words and phrases. Menger space, Hurewicz space, $\gamma$-space, filter, ideal, Mathias forcing.

${ }^{1}$ While completing this manuscript we have learned that Corollary 1.2 for Borel filters has been independently obtained in [11]. 
Corollary 1.2. Let $\mathcal{F}$ be an analytic filter on $\omega$. Then $\mathbb{M}_{\mathcal{F}}$ does not add a dominating real if and only if $\mathcal{F}$ is $\sigma$-compact.

Several additional applications of Theorem 1.1 will be presented in Section 3 .

Following [10] we say that a family $\mathcal{U}$ of subsets of a set $X$ is

- an $\omega$-cover, if $X \notin \mathcal{U}$ and for every finite subset $K$ of $X$ there exists $U \in \mathcal{U}$ such that $K \subset U$;

- a $\gamma$-cover, if for every $x \in X$ the family $\{U \in \mathcal{U}: x \notin U\}$ is finite.

The Hurewicz (resp. Scheepers ${ }^{2}$ ) property is defined in the same way as the Menger one, the only difference being that the family $\left\{\bigcup \mathcal{V}_{n}: n \in \omega\right\}$ must be a $\gamma$-cover (resp. $\omega$-cover) of $X$.

We say that a poset $\mathbb{P}$ is almost $\omega^{\omega}$-bounding if for every $\mathbb{P}$-name $\dot{f}$ for a real and $q \in \mathbb{P}$, there exists $g: \omega \rightarrow \omega$ such that for every $A \in[\omega]^{\omega}$ there is $q_{A} \leq q$ such that $q_{A} \Vdash g\left\lceil A \nless^{*} \dot{f} \uparrow A\right.$. It is well known that almost $\omega^{\omega}$ bounding posets preserve unbounded families of reals of the ground model as unbounded families in the generic extension. This was observed by Shelah in [22]. The following lemma shows that this property in fact characterizes almost $\omega^{\omega}$-bounding posets. We are not aware of this fact having been mentioned in the literature before.

Lemma 1.3. A poset $\mathbb{P}$ is almost $\omega^{\omega}$-bounding if and only if $\mathbb{P}$ preserves all unbounded families of the ground model as unbounded families in the extension.

Proof. Suppose that $\mathbb{P}$ is not almost $\omega^{\omega}$-bounding. There is a name $\dot{f}$ for a real and $p \in \mathbb{P}$, a condition such that for all $g \in \omega^{\omega}$ there is an infinite set $A_{g} \in[\omega]^{\omega}$ such that $p \Vdash g \uparrow A_{g} \leq^{*} \dot{f} \uparrow A_{g}$. For every $g \in \omega^{\omega}$ define $g^{\prime}(n)=g(n)$ if $n \in A_{g}$, and $g^{\prime}(n)=0$ otherwise. The set $X=\left\{g^{\prime}: g \in \omega^{\omega}\right\}$ is an unbounded set of reals, and the condition $p$ forces $X$ to be bounded by $\dot{f}$ in the extension.

Suppose that $\mathbb{P}$ is almost $\omega^{\omega}$-bounding and let $X$ be an unbounded set of reals. Let $\dot{f}$ be a name for a real and $q \in \mathbb{P}$ a condition. Find $g: \omega \rightarrow \omega$ as in the definition of an almost $\omega^{\omega}$-bounding forcing. Since $X$ is unbounded, there is $h \in X$ such that $A=\{n \in \omega: g(n)<h(n)\}$ is infinite. Now $q_{A} \leq q$ forces $h$ to be not dominated by $\dot{f}$.

The following theorem is the main result of section 4 .

Theorem 1.4. Let $\mathcal{F}$ be a filter. Then $\mathbb{M}_{\mathcal{F}}$ is almost $\omega^{\omega}$-bounding if and only if $\mathcal{F}$ has the Hurewicz property.

Theorem 1.4 turns out to have applications to general Hurewicz spaces, not only to filters. In order to formulate them we need to recall some definitions. A Tychonov space $X$ is called a $\gamma$-space [10] if every open $\omega$ cover of $X$ contains a $\gamma$-subcover. $\gamma$-spaces are important in the theory of function spaces as they are exactly those $X$ for which the space $C_{p}(X)$ of continuous functions from $X$ to $\mathbb{R}$, with the topology inherited from $\mathbb{R}^{X}$, has the Fréchet-Urysohn property.

\footnotetext{
${ }^{2}$ In $[20]$ this property is denoted by $U_{f i n}(\mathcal{O}, \Omega)$. The name "Scheepers property" was suggested by Banakh and by now seems to have become quite standard.
} 
For $a \in[\omega]^{\omega}$ and $n \in \omega, a(n)$ denotes the $n$-th element in the increasing enumeration of $a$. For $a, b \in[\omega]^{\omega}, a \leq^{*} b$ means that $a(n) \leq b(n)$ for all but finitely many $n$. A $\mathfrak{b}$-scale is an unbounded set $S=\left\{s_{\alpha}: \alpha<\mathfrak{b}\right\}$ in $\left([\omega]^{\omega}, \leq^{*}\right)$ such that $s_{\alpha} \leq^{*} s_{\beta}$ for $\alpha<\beta$. It is easy to see that $\mathfrak{b}$-scales exist in ZFC. For each $\mathfrak{b}$-scale $S, S \cup[\omega]^{<\omega}$ is $\mathfrak{b}$-concentrated on $[\omega]^{<\omega}$ in the sense that $|S \backslash U|<\mathfrak{b}$ for any open $U \supset[\omega]^{<\omega}$. For brevity, the union of a $\mathfrak{b}$-scale with $[\omega]^{<\omega}$, viewed as a subset of the Cantor space $\mathcal{P}(\omega)$, will be called $a$ $\mathfrak{b}$-scale set. As an application of Theorem 1.4 we will get the following result answering $[19$, Problem 4.2] in the affirmative.

Corollary 1.5. It is consistent with $Z F C$ that every $\mathfrak{b}$-scale set is a $\gamma$-space.

The study of the relation between $\mathfrak{b}$-scale sets and $\gamma$-spaces already has some history. First of all, in the Laver model all $\gamma$-subspaces of $2^{\omega}$ are countable because they have strong measure zero [10]. Answering one of the questions posed in [10], Galvin and Miller [9] constructed under $\mathfrak{p}=$ $\mathfrak{c}$ a $\mathfrak{b}$-scale set which is a $\gamma$-set. Their $\mathfrak{b}$-scale was a tower, where $S=$ $\left\{s_{\alpha}: \alpha<\kappa\right\} \subset[\omega]^{\omega}$ is called a tower if $s_{\alpha} \subset^{*} s_{\beta}$ for all $\beta<\alpha$ and $S$ has no pseudointersection. Later Orenshtein and Tsaban proved [18] that if $\mathfrak{p}=\mathfrak{b}$ then any $\mathfrak{b}$-scale set is a $\gamma$-space provided that the corresponding $\mathfrak{b}$-scale is a tower. On the other hand, under $\mathfrak{b}=\mathfrak{c}$ there exists a $\mathfrak{b}$-scale set which fails to be a $\gamma$-space, see [19]. Also, it is easy to show that such $\mathfrak{b}$-scale sets exist under $\mathfrak{p}<\mathfrak{b}$, see Observation 4.4. Thus $\mathfrak{p}=\mathfrak{b}<\mathfrak{c}$ holds in any model of Corollary 1.5.

In section 5 we discuss when an unbounded subset of $\omega^{\omega}$ can be made bounded by forcing without introducing dominating reals. Some partial answers are give for filters, see Theorem 5.2 and the Remark 5.6 at the end of the section.

While dealing with the covering properties of Menger, Hurewicz, and Scheepers, as well as that of being the $\gamma$-space, we shall freely use that they are (as almost all natural covering properties) inherited by continuous images and closed subspaces. In addition, the properties of Menger and Hurewicz are preserved by products with $\sigma$-compact spaces and by countable unions. These straightforward facts exist in the literature, but we do not give any references because we believe that the reader will need just a couple of minutes to check any of them.

For the definitions of cardinal characteristics used in this paper we refer the reader to [25].

\section{Proof of Theorem 1.1}

Subsets of $\mathcal{P}(\omega)$ are considered as usual with the topology inherited from $\mathcal{P}(\omega)$, which is identified with the Cantor space $2^{\omega}$ via characteristic functions. For every $n \in \omega$ and $q \subset n$ we denote the set $\{A \in \mathcal{P}(\omega): A \cap n=q\}$ by $[n, q]$. The sets of the form $[n, q]$ form a base for the standard topology of $\mathcal{P}(\omega)$. Set also $\uparrow X=\{A \in \mathcal{P}(\omega): A \supset X\}$ for every $X \subset \omega$.

Claim 2.1. Suppose that $\mathcal{X} \subset \mathcal{P}(\omega)$ is closed under taking supersets and $\mathcal{O}$ is a cover of $\mathcal{X}$ by sets open in $\mathcal{P}(\omega)$. Then there exists a family $Q \subset[\omega]<\omega$ 
such that

$$
\mathcal{X} \subset \bigcup_{q \in Q} \uparrow q \subset \bigcup \mathcal{O} .
$$

Proof. Without loss of generality, we can assume that $\mathcal{O}$ consists of sets of the form $[n, q]$. Let us fix $X \in \mathcal{X}$, notice that $\uparrow X$ is compact, and find a finite family of basic open sets $\left\{\left[n_{i}, q_{i}\right]: i \in m\right\} \subset \mathcal{O}$ such that $\uparrow X \subset \bigcup_{i \in m}\left[n_{i}, q_{i}\right]$. Put $n=\max \left\{n_{i}: i \in m\right\}$. If $A \in \uparrow(X \cap n)$, then $(A \cap n) \cup(\omega \backslash n) \in \uparrow X$, and there is $i \in m$ such that $(A \cap n) \cup(\omega \backslash n) \in\left[n_{i}, q_{i}\right]$; thus $A \in\left[n_{i}, q_{i}\right]$. We showed that $X \in \uparrow X \subset \uparrow(X \cap n) \subset \bigcup \mathcal{O}$.

Since every set of the form $\uparrow q$ is compact, it follows that for every $q \in Q$ (we use notation from Claim 2.1) there exists a finite subset $\mathcal{O}^{\prime} \subset \mathcal{O}$ such that $\uparrow q \subset \cup \mathcal{O}^{\prime}$. This gives us the following

Corollary 2.2. If $\mathcal{X} \subset \mathcal{P}(\omega)$ is closed under taking supersets, then $\mathcal{X}$ has the Menger property if and only if for every sequence $\left\langle\mathcal{U}_{n}: n \in \omega\right\rangle$ of open covers of $\mathcal{X}$ by sets of the form $\uparrow q$ for some $q \in[\omega]^{<\omega}$, there exists a sequence $\left\langle\mathcal{V}_{n}: n \in \omega\right\rangle$ such that $\mathcal{V}_{n} \in\left[\mathcal{U}_{n}\right]^{<\omega}$ and $\left\{\bigcup \mathcal{V}_{n}: n \in \omega\right\}$ is a cover of $\mathcal{X}$.

A set $\mathcal{I} \subset \mathcal{P}(\omega)$ is called an ideal, if $\mathcal{F}:=\{\omega \backslash I: I \in \mathcal{I}\}$ is a filter. In this case we write $\mathcal{I}=\mathcal{F}^{*}$ and $\mathcal{F}=\mathcal{I}^{*}$. The collection of all $\mathcal{I}$-positive sets $\mathcal{P}(\omega) \backslash \mathcal{I}$ is denoted by $\mathcal{I}^{+}$or $\mathcal{F}^{+}$, if $\mathcal{F}$ is the filter dual to $\mathcal{I}$. Following [12] we call an ideal $\mathcal{I}$ a $P^{+}$-ideal if for every decreasing sequence $\left\langle X_{n}: n \in \omega\right\rangle$ of $\mathcal{I}$-positive sets, there is an $X \in \mathcal{I}^{+}$such that $X \subset^{*} X_{n}$ for all $n \in \omega$. We shall also use the following notation:

$$
\mathcal{I}^{<\omega}=\left\{A \subset[\omega]^{<\omega}: \exists I \in \mathcal{I} \forall a \in A(a \cap I \neq \emptyset)\right\} .
$$

It is easy to see that $\mathcal{I}^{<\omega}$ is an ideal on $[\omega]^{<\omega}$, and letting $\mathcal{F}=\mathcal{I}^{*}$ we have $\mathcal{I}^{<\omega}=\left(\mathcal{F}^{<\omega}\right)^{*}$, where $\mathcal{F}^{<\omega}$ is the filter on $[\omega]^{<\omega}$ consisting of sets containing $[F]<\omega$ for some $F \in \mathcal{F}$.

The following claim is known, we give here a proof for reader's convenience. A stronger form of this result is presented in [17].

Claim 2.3. Let $\mathcal{I}$ be an ideal on $\omega$. Then $\mathcal{I}$ is a $P^{+}$-ideal if and only if for every sequence $\left\langle X_{n}: n \in \omega\right\rangle$ of $\mathcal{I}$-positive sets there is a sequence $\left\langle Y_{n}: n \in \omega\right\rangle$ of finite sets such that $Y_{n} \subset X_{n}$ and $\bigcup_{n \in \omega} Y_{n} \in \mathcal{I}^{+}$.

Proof. The "if" part is obvious. To prove the "only if" part fix a sequence $\left\langle X_{n}: n \in \omega\right\rangle$ of $\mathcal{I}$-positive sets and set $X_{n}^{\prime}=\bigcup_{m>n} X_{m}$ for all $n \in \omega$. Then $\left\langle X_{n}^{\prime}: n \in \omega\right\rangle$ is a decreasing sequence of $\mathcal{I}$-positive sets, and hence there exists $Y \in \mathcal{I}^{+}$such that $Y \subset^{*} X_{n}^{\prime}$ for all $n \in \omega$. Without loss of generality we may assume that $Y \subset X_{0}^{\prime}$. For every $y \in Y \backslash \bigcap_{n \in \omega} X_{n}^{\prime}$ let $n(y)$ be the maximal $n$ such that $y \in X_{n}^{\prime}$. For $y \in Y \cap \bigcap_{n \in \omega} X_{n}^{\prime}$ let $n(y)$ be any $n>y$. Then $Y_{n}=\{y \in Y: n(y)=n\}$ is finite, $Y_{n} \subset X_{n}$, and $Y=\bigcup_{n \in \omega} Y_{n}$.

The proof of the following fact is more or less just a reformulation.

Claim 2.4. Let $\mathcal{I}$ be an ideal. Then $\mathcal{I}^{<\omega}$ is a $P^{+}$-ideal if and only if $\mathcal{I}$ is a Menger subspace of $\mathcal{P}(\omega)$.

Proof. Since $\mathcal{I}$ is homeomorphic to $\mathcal{I}^{*}$ it is enough to show that $\mathcal{I}^{*}$ is Menger if and only if $\mathcal{I}^{<\omega}$ is a $P^{+}$-ideal. 
Assume that $\mathcal{I}^{<\omega}$ is a $P^{+}$-ideal and fix a sequence $\left\langle\mathcal{U}_{n}: n \in \omega\right\rangle$ of open covers of $\mathcal{I}^{*}$ by sets of the form $\uparrow a$ for some $a \in[\omega]^{<\omega}$. Set $A_{n}=\{a: \uparrow a \in$ $\left.\mathcal{U}_{n}\right\}$. Since $\mathcal{U}_{n}$ covers $\mathcal{I}^{*}$, for every $F \in \mathcal{I}^{*}$ there exists $a \in A_{n}$ such that $a \subset F$, which means that $A_{n}$ is $\mathcal{I}^{<\omega}$-positive. Therefore there exists a sequence $\left\langle B_{n}: n \in \omega\right\rangle$ such that $B_{n} \in\left[A_{n}\right]^{<\omega}$ and $B=\bigcup_{n \in \omega} B_{n} \in\left(\mathcal{I}^{<\omega}\right)^{+}$. This means that for every $F \in \mathcal{I}^{*}$ there exists $b \in B$ such that $b \subset F$, i.e., that $\{\uparrow b: b \in B\}$ covers $\mathcal{I}^{*}$. Thus for every $n$ we can select a finite subset of $\mathcal{U}_{n}$ (namely $\mathcal{V}_{n}=\left\{\uparrow b: b \in B_{n}\right\}$ ) whose union covers $\mathcal{I}^{*}$. By Corollary 2.2 this means that $\mathcal{I}^{*}$ is Menger.

Now suppose that $\mathcal{I}^{*}$ is Menger and fix a sequence $\left\langle A_{n}: n \in \omega\right\rangle$ of $\mathcal{I}^{<\omega}$ positive sets. For every $n$ set $\mathcal{U}_{n}=\left\{\uparrow a: a \in A_{n}\right\}$ and notice that $\mathcal{U}_{n}$ is a cover of $\mathcal{I}^{*}$ by sets open in $\mathcal{P}(\omega)$. Thus for every $n$ there exists a finite $\mathcal{V}_{n} \subset \mathcal{U}_{n}$ such that $\bigcup_{n \in \omega} \mathcal{V}_{n} \supset \mathcal{I}^{*}$. Let $B_{n} \in\left[A_{n}\right]^{<\omega}$ be such that $\mathcal{V}_{n}=$ $\left\{\uparrow a: a \in B_{n}\right\}$. It follows that for every $F \in \mathcal{I}^{*}$ there exists $a \in \bigcup_{n \in \omega} B_{n}$ such that $a \subset F$. In other words, $\bigcup_{n \in \omega} B_{n}$ is $\mathcal{I}^{<\omega}$-positive, which completes our proof.

Now Theorem 1.1 is a direct consequence of Claim 2.4, the fact that $\mathcal{F}$ is homeomorphic to $\mathcal{F}^{*}$ for any filter $\mathcal{F}$, and the following important

Theorem 2.5. [12, Theorem 3.8] Let $\mathcal{I}$ be an ideal on $\omega$. Then $\mathbb{M}_{\mathcal{I}^{*}}$ does not add a dominating real if and only if $\mathcal{I}^{<\omega}$ is a $P^{+}$-ideal.

\section{Straightforward applications of Theorem 1.1}

Recall that $A, B$ are called almost disjoint, if $A \cap B$ is finite. Given a countable set $I$, an infinite set $\mathcal{A} \subset[I]^{\omega}$ is said to be an almost disjoint family (on $I$ ) if any two elements of $\mathcal{A}$ are almost disjoint. $\mathcal{A}$ is called a mad family (on $I$ ), if it is maximal with respect to inclusion among almost disjoint families on $I$. Every almost disjoint family $\mathcal{A}$ generates an ideal

$$
\mathcal{I}(\mathcal{A})=\left\{I \subset \omega: \exists \mathcal{B} \in[\mathcal{A}]^{<\omega}\left(I \subset^{*} \bigcup \mathcal{B}\right)\right\} .
$$

The dual filter is denoted by $\mathcal{F}(\mathcal{A})$. Theorem 1.1 allows us to give an easy proof of the following recent result of Guzmán, Hrušák and Martínez [11, Proposition 6], answering [6, Question 2.7] in the negative.

Proposition 3.1. There exists a mad family $\mathcal{A}$ on $\omega$ such that $\mathbb{M}_{\mathcal{F}(\mathcal{A})}$ adds a dominating real.

Proof. By Theorem 1.1 it is enough to construct a mad family $\mathcal{A}$ on $2^{<\omega}$ such that $\mathcal{I}(\mathcal{A})$ is not Menger. Set $\mathcal{C}=\left\{C_{x}: x \in 2^{\omega}\right\}$, where $C_{x}=\{x\lceil n: n \in \omega\}$. Then $\mathcal{C}$ is a compact almost disjoint family. Take a dense countable subset $\mathcal{C}^{\prime}$ of $\mathcal{C}$ and for every $C$ in $\mathcal{C}^{\prime}$ fix an infinite mad family $\mathcal{A}_{C}$ of infinite subsets of $C$. Consider $\mathcal{A}_{0}=\left(\mathcal{C} \backslash \mathcal{C}^{\prime}\right) \cup \bigcup_{C \in \mathcal{C}^{\prime}} \mathcal{A}_{C}$ and extend $\mathcal{A}_{0}$ to a mad family $\mathcal{A}$ of infinite subsets of $2^{<\omega}$.

We claim that $C \backslash \bigcup \mathcal{B}$ is infinite for all $\mathcal{B} \in[\mathcal{A}]^{<\omega}$ and $C \in \mathcal{C}^{\prime}$. Indeed, let us fix $C, \mathcal{B}$, and $A \in \mathcal{A}_{C} \backslash \mathcal{B}$. Then all elements of $\mathcal{B}$ have finite intersection with $A$ and hence $A \not \subset^{*} \cup \mathcal{B}$. Therefore $C \not \subset^{*} \cup \mathcal{B}$ as well.

Thus $\mathcal{I}(\mathcal{A}) \cap \mathcal{C}=\mathcal{C} \backslash \mathcal{C}^{\prime}$, and hence $\mathcal{I}(\mathcal{A})$ contains a closed copy of $\omega^{\omega}$. It remains to note that $\omega^{\omega}$ is not Menger and that the Menger property is inherited by closed subsets. 
As mentioned before, the Menger and Hurewicz properties are preserved by closed subspaces and products with compact spaces, continuous images, and countable unions. Thus if a filter $\mathcal{F}$ on $\omega$ has a base $\mathcal{B}$ which is Menger (Hurewicz), then $\mathcal{F}$ is Menger (Hurewicz) as well: $\mathcal{F}=\psi[\mathcal{B} \times \mathcal{P}(\omega)]$, where $\psi(B, X)=B \cup X$, and $\psi$ is continuous.

Let $\mathcal{U}$ be an ultrafilter. For $x, y \in \omega^{\omega}$ the notation $x \leq \mathcal{U} y$ means that $\{n: x(n) \leq y(n)\} \in \mathcal{U}$. We will also use the notation $A \leq \mathcal{U} B$ for $A, B \in[\omega]^{\omega}$ by interpreting sets as their enumerating functions. The relation $\leq_{\mathcal{U}}$ is a linear pre-ordering of $\omega^{\omega}$ whose cofinality is usually denoted by $\mathfrak{d}(\mathcal{U})$.

Another application of Theorem 1.1 is the following result improving [11, Proposition 8] and partially answering [11, Problem 2]. Instead of proving it directly we shall give a more streamlined argument using [11, Proposition 8].

Proposition 3.2. If $\mathfrak{d}=\mathfrak{c}$, then there exists an infinite mad family $\mathcal{A}$ such that $\mathcal{I}(\mathcal{A})$ is Menger.

Proof. First assume that $\mathfrak{d}$ is regular and fix an enumeration $\left\{S_{\alpha}: \alpha<\mathfrak{d}\right\}$ of $[\omega]^{\omega}$ such that $\left\{S_{\alpha}: \alpha<\omega\right\}$ is an almost disjoint family. It is well-known [8] that there exists an ultrafilter $\mathcal{U}$ with $\mathfrak{d}(\mathcal{U})=\operatorname{cf}(\mathfrak{d})$, which equals $\mathfrak{c}$ in our case. We shall construct $\mathcal{A}=\left\{A_{\alpha}: \alpha<\mathfrak{d}\right\}$ by induction. At stage $\alpha$ we pick $A_{\alpha} \subset S_{\alpha}$ such that $\left\{S_{\beta}: \beta<\alpha\right\} \cup\left\{A_{\beta}: \beta<\alpha\right\} \leq_{\mathcal{U}} A_{\alpha}$ provided that $S_{\alpha}$ is almost disjoint from $A_{\beta}$ for all $\beta<\alpha$. Otherwise we set $A_{\alpha}$ to be equal to one of the $A_{\beta}$ 's constructed before. This finishes our construction of $\mathcal{A}$. It is clear that $\mathcal{A}$ is mad. It is well known and easy to see that $\mathfrak{d}$ is the minimal cardinality of non-Menger set of reals. Hence if $|\mathcal{A}|<\mathfrak{d}$, then all finite powers of $\mathcal{A}$ are Menger. If $|\mathcal{A}|=\mathfrak{d}$ then [24, Cor. 4.3] ensures that all finite powers of $\mathcal{A} \cup[\omega]^{<\omega}$ are Menger. In any case, $\mathcal{I}(\mathcal{A})$ is Menger because it can be written in the form $\bigcup_{n \in \omega} \mathcal{I}_{n}$, where

$$
\mathcal{I}_{n}=\left\{\bigcup_{i \in n} A^{i} \cap X:\left\langle A^{i}: i \in n\right\rangle \in\left(\mathcal{A} \cup[\omega]^{<\omega}\right)^{n}, X \in \mathcal{P}(\omega)\right\}
$$

is a continuous image of $\left(\mathcal{A} \cup[\omega]^{<\omega}\right)^{n} \times \mathcal{P}(\omega)$.

Now suppose that $\mathfrak{d}$ is singular. It has been established in the proof of [5, Theorem 16] that $\mathfrak{u}<\mathfrak{d}$ yields $\mathfrak{d}(\mathcal{U})=\mathfrak{d}$ for any ultrafilter $\mathcal{U}$ generated by $\mathfrak{u}$ many sets. Thus $\mathfrak{u}<\mathfrak{d}$ implies that $\mathfrak{d}$ is regular, and hence we have $\mathfrak{u}=\mathfrak{d}=\mathfrak{c}$ by the singularity of $\mathfrak{d}$. Now $\min \{\mathfrak{d}, \mathfrak{u}\} \leq \mathfrak{r}$ (see [2]) implies $\mathfrak{r}=\mathfrak{c}$ and it suffices to apply [11, Prop. 8] which states that under $\mathfrak{d}=\mathfrak{r}=\mathfrak{c}$ there exists a mad family generating a Menger ideal.

Observation 3.3. If a filter $\mathcal{F}$ is Menger (Hurewicz), then so is $\mathcal{F}^{<\omega}$.

Note that the converse implication is also true since $\mathcal{F}$ is isomorphic to a closed subset of $\mathcal{F}^{<\omega}$.

Proof. The map $\phi: \mathcal{F} \rightarrow \mathcal{P}\left([\omega]^{<\omega}\right)$ assigning to $F \in \mathcal{F}$ the set $[F]^{<\omega}$ is continuous. Thus $\mathcal{F}^{<\omega}$ has a Menger (Hurewicz) base and hence is Menger (Hurewicz).

Combining Theorem 1.1, Observation 3.3, and [11, Prop. 5] we get a negative answer to $[11 \text {, Problem } 4]^{3}$.

\footnotetext{
${ }^{3}$ The formulation of [11, Problem 4] involves notions which will not be used in our paper, and hence we refer the reader to [11] for its precise formulation.
} 
Following [11] (Laflamme [16] for ultrafilters) we say that a filter $\mathcal{F}$ is a strong $P^{+}$-filter if for every sequence $\left\langle\mathcal{C}_{n}: n \in \omega\right\rangle$ of compact subsets of $\mathcal{F}^{+}$there exists an increasing sequence $\left\langle k_{n}: n \in \omega\right\rangle$ of integers such that if $X_{n} \in \mathcal{C}_{n}$ for all $n$, then $\bigcup_{n \in \omega}\left(X_{n} \cap\left[k_{n}, k_{n+1}\right)\right) \in \mathcal{F}^{+}$. The characterization of $P^{+}$-filters given in Claim 2.3 implies that every strong $P^{+}$-filter is a $P^{+}$filter.

We shall need the following game of length $\omega$ on a topological space $X$ : In the $n$th move player $I$ chooses a countable open cover $\mathcal{U}_{n}$ of $X$, and player II responds by choosing a finite $\mathcal{V}_{n} \subset \mathcal{U}_{n}$. Player II wins the game if $\bigcup_{n \in \omega} \cup \mathcal{V}_{n}=X$. Otherwise, player $I$ wins. We shall call this game the Menger game $e^{4}$ on $X$. It is well-known that $X$ is Menger if and only if player $I$ has no winning strategy in the Menger game on $X$, see [13] or [20, Theorem 13]. Note that if $I$ plays with covers $\mathcal{U}_{n}$ closed under finite unions, then we can assume that the player II replies by choosing oneelement subsets of the $\mathcal{U}_{n}$ 's.

The following result together with [11, Proposition 3] answers [11, Problem 3] in the negative.

Proposition 3.4. Every Menger filter $\mathcal{F}$ is a strong $P^{+}$-filter.

Proof. Let $\left\langle\mathcal{C}_{n}: n \in \omega\right\rangle$ be a sequence of compact subsets of $\mathcal{F}^{+}$, and assume without loss of generality that $\mathcal{C}_{n} \subseteq \mathcal{C}_{m}$ for $n<m$. For every $F \in \mathcal{F}$ consider an increasing sequence $\left\langle k_{n}^{F}: n \in \omega\right\rangle$ defined as follows: $k_{0}^{F}=0$, and $k_{n+1}^{F}$ is the minimal integer such that $\left[k_{n}^{F}, k_{n+1}^{F}\right) \cap F \cap X \neq \emptyset$ for all $X \in \mathcal{C}_{n}$. The existence of such a number follows by the compactness of $\mathcal{C}_{n}$. Moreover, it is easy to see that $F \mapsto\left\langle k_{n}^{F}: n \in \omega\right\rangle$ is a continuous map from $\mathcal{F}$ to $\omega^{\omega}$, and hence its range $K:=\left\{\left\langle k_{n}^{F}\right\rangle_{n \in \omega}: F \in \mathcal{F}\right\} \subset \omega^{\omega}$ is Menger.

Let us consider the following strategy of the player $I$ in the Menger game on $K: I$ starts by choosing the cover $\mathcal{U}_{0}=\left\{U_{m}^{0}: m \in \omega\right\}$ of $K$, where $U_{m}^{0}$ is the set of all $\left\langle k_{n}^{F}\right\rangle_{n \in \omega} \in K$ such that $k_{1}^{F}<m$. Suppose that II replies by choosing $U_{k_{0}}^{0}$. Then in the next move $I$ chooses a cover $\mathcal{U}_{1}=\left\{U_{m}^{1}: m \in\right.$ $\left.\omega, m>k_{0}\right\}$ of $K$, where $U_{m}^{1}$ is the set of all $\left\langle k_{n}^{F}\right\rangle_{n \in \omega} \in K$ such that $k_{k_{0}+1}^{F}<$ $m$. If $I I$ replies by choosing $U_{k_{1}}^{1}$, then $I$ chooses $\mathcal{U}_{2}=\left\{U_{m}^{2}: m \in \omega, m>k_{1}\right\}$, where $U_{m}^{2}$ is the set of all $\left\langle k_{n}^{F}\right\rangle_{n \in \omega} \in K$ such that $k_{k_{1}+1}^{F}<m$, and so on. Since $K$ is Menger, the strategy of $I$ defined above is not winning. Therefore there exists a play $\left\langle\mathcal{U}_{n}, U_{k_{n}}^{n}: n \in \omega\right\rangle$ in which $I$ follows this strategy and looses, i.e., $K \subset \bigcup_{n \in \omega} U_{k_{n}}^{n}$. We claim that the sequence $\left\langle k_{n}: n \in \omega\right\rangle$ is as required. For this we shall show that for any $F \in \mathcal{F}$ and any sequence $\left\langle X_{n} \in \mathcal{C}_{n}: n \in \omega\right\rangle$ there exists $n$ such that $X_{n} \cap F \cap\left[k_{n}, k_{n+1}\right) \neq \emptyset$. Indeed, since $K \subset \bigcup_{n \in \omega} U_{k_{n}}^{n}$, it follows that there exists $n$ such that $k_{n} \leq k_{k_{n}}^{F}<k_{k_{n}+1}^{F}<k_{n+1}$. Since $X_{n} \in \mathcal{C}_{n} \subseteq \mathcal{C}_{k_{n}}$, we have that $\left[k_{k_{n}}^{F}, k_{k_{n}+1}^{F}\right) \cap F \cap X_{n} \neq \emptyset$, which completes our proof.

To conclude this section, let us review various equivalent characterizations of filters $\mathcal{F}$ for which the forcing $\mathbb{M}_{\mathcal{F}}$ does not add dominating reals. The following theorem combines results of this paper with results from [11].

\footnotetext{
${ }^{4}$ In case $X$ is a filter on $\omega$, notice the similarity of this game to the game $\mathfrak{G}\left(\mathcal{F}^{+},[\omega]^{<\omega}, \mathcal{F}^{+}\right)$from [17], where $\mathcal{F}=X^{<\omega}$.
} 
Theorem 3.5. Let $\mathcal{F}$ be a filter on $\omega$. The following are equivalent:

(1) $\mathbb{M}_{\mathcal{F}}$ does not add dominating reals,

(2) $\mathcal{F}$ is Menger,

(3) $\mathcal{F}^{<\omega}$ is Menger,

(4) $\mathcal{F}^{<\omega}$ is a $P^{+}$filter,

(5) $\mathcal{F}$ is a strong $P^{+}$filter,

(6) $\mathcal{F}^{<\omega}$ is a strong $P^{+}$filter.

\section{HuREwicz FILTERS AND $\gamma$-SPACES}

First we shall prove Theorem 1.4. Suppose that $\mathcal{F}$ is Hurewicz, but there exists an unbounded $X \subset \omega^{\omega}, X \in V$, and an $\mathbb{M}_{\mathcal{F}}$-name $\dot{g}$ for a function dominating $X$ (for simplicity assume that every condition forces this). For every $x \in X$ let us find $n^{x} \in \omega$ and a condition $\left\langle s^{x}, F^{x}\right\rangle$ forcing $x(n)<\dot{g}(n)$ for all $n \geq n^{x}$. Since $X$ cannot be covered by a countable family of bounded sets, we may assume that $s^{x}$ and $n^{x}$ do not depend on $x$, i.e., $s^{x}=s_{*}$ and $n^{x}=n_{*}$ for all $x \in X$.

For every $m \in \omega$ let $\mathcal{S}_{m}$ be the set of those $s \in[\omega]^{<\omega}$ such that max $s_{*}<$ min $s$ and there exist $F_{s} \in \mathcal{F}$ such that $\left\langle s_{*} \cup s, F_{s}\right\rangle$ forces $\dot{g}(m)$ to be equal to some $g_{s}(m)$. It is clear that for every $F \in \mathcal{F}$ there exists $s \in \mathcal{S}_{m}$ such that $s \subset F$. In other words, $\mathcal{U}_{m}:=\left\{\uparrow s: s \in \mathcal{S}_{m}\right\}$ is an open cover of $\mathcal{F}$. Since $\mathcal{F}$ is Hurewicz, for every $m$ there exists a finite $\mathcal{V}_{m} \subset \mathcal{U}_{m}$ such that $\left\{\bigcup \mathcal{V}_{m}: m \in \omega\right\}$ is a $\gamma$-cover of $\mathcal{F}$. Let $\mathcal{T}_{m} \in\left[\mathcal{S}_{m}\right]^{<\omega}$ be such that $\mathcal{V}_{m}=\left\{\uparrow s: s \in \mathcal{T}_{m}\right\}$ and $f(m)=\max \left\{g_{s}(m): s \in \mathcal{T}_{m}\right\}$. We will derive a contradiction by showing that $x<^{*} f$ for each $x \in X$. Fix $x \in X$ and $l \in \omega$ such that for every $m \geq l$ there exists $s_{m} \in \mathcal{T}_{m}$ such that $F^{x} \in \uparrow s_{m}$. Pick any $m \geq n_{*}, l$. Since $\left\langle s_{*}, F^{x}\right\rangle \Vdash x(m)<\dot{g}(m),\left\langle s_{*} \cup s_{m}, F_{s_{m}}\right\rangle \Vdash \dot{g}(m) \leq f(m)$, and these two conditions are compatible, it follows that $x(m)<f(m)$.

Now suppose that $\mathcal{F}$ is not Hurewicz as witnessed by a sequence $\left\langle\mathcal{U}_{n}: n \in\right.$ $\omega)$ of covers of $\mathcal{F}$ by sets open in $\mathcal{P}(\omega)$. By Claim 2.1 we may additionally assume that $\mathcal{U}_{n}=\left\{\uparrow q_{m}(n): m \in \omega\right\}$, where $q_{m}(n) \in[\omega]^{<\omega}$. For every $F \in \mathcal{F}$ consider the function $x_{F} \in \omega^{\omega}, x_{F}(n)=\min \left\{m: F \in \uparrow q_{m}(n)\right\}$. It follows from the fact that $\mathcal{F}$ is not Hurewicz that $X=\left\{x_{F}: F \in \mathcal{F}\right\}$ is unbounded.

Now let $G$ be the generic pseudointersection of $\mathcal{F}$ added by $\mathbb{M}_{\mathcal{F}}$.

Claim 4.1. For every $n$ there exists $g(n)$ such that $G \backslash n \in \uparrow q_{g(n)}(n)$.

Proof. The set $\mathcal{U}_{n}^{\prime}=\left\{\uparrow q_{m}(n): q_{m}(n) \cap n=\emptyset, m \in \omega\right\}$ covers $\mathcal{F}$ because $\mathcal{U}_{n}$ is a cover of $\mathcal{F}$. Hence for every $F \in \mathcal{F}$ there is some $\uparrow q_{m}(n) \in \mathcal{U}_{n}^{\prime}$ such that $F \in \uparrow q_{m}(n)$, and the set of conditions $\langle s, F\rangle$ such that $q_{m}(n) \subseteq s \backslash n$ for some $m \in \omega$ is dense.

Let us fix $F \in \mathcal{F}$ and find $n$ such that $G \backslash n \subset F$. Then $G \backslash n \in$ $\uparrow q_{g(n)}(n)$ yields $F \in \uparrow q_{g(n)}(n)$, which implies $x_{F}(n) \leq g(n)$. Thus $g \in \omega^{\omega}$ is dominating $X$, and therefore $\mathbb{M}_{\mathcal{F}}$ fails to preserve ground model unbounded sets.

$\square_{\text {Theorem } 1.4}$

Remark 4.2. Theorem 1.1 can be proved directly using the ideas of the proof of Theorem 1.4. On the other hand, the proof of [12, Theorem 3.8] could be easily modified to get a combinatorial characterization of filters $\mathcal{F}$ such that $\mathbb{M}_{\mathcal{F}}$ is almost $\omega^{\omega}$-bounding, and then Theorem 1.4 can be 
proved in the same way as Theorem 1.1. We have deliberately presented two approaches.

By $[4$, Theorem 10] every $\mathfrak{b}$-scale set has the Hurewicz property in all finite powers. Thus Corollary 1.5 is a direct consequence of the following

Theorem 4.3. It is consistent with $Z F C$ that $\mathfrak{b}=\omega_{1}$ and every Tychonov space $X$ of size $\omega_{1}$ is a $\gamma$-space provided that $X^{n}$ is Hurewicz for all $n \in \omega$.

Proof. Using Theorem 1.4, a standard book-keeping argument taking care of all filters $\mathcal{F}$ on $\omega$ having a Hurewicz base $\mathcal{B}$ of size $\omega_{1}$, and the well-known fact that unbounded well-ordered by $\leq^{*}$ subfamilies of $\omega^{\omega}$ are preserved at limit stages of finite support iterations of c.c.c. posets (see, e.g., [3, Lemma 6.5.7]), we can perform an $\omega_{2}$ steps finite support iteration $\mathbb{P}_{\omega_{2}}=\left\langle\mathbb{P}_{\alpha}, \dot{\mathbb{Q}}_{\alpha}: \alpha<\omega_{2}\right\rangle$ of c.c.c. posets such that in $V^{\mathbb{P}_{\omega_{2}}}$ the following holds:

(i) $\mathfrak{b}=\omega_{1}$;

(ii) Every filter $\mathcal{F}$ on $\omega$ has a pseudointersection provided it has a Hurewicz base $\mathcal{B}$ of size $\omega_{1}$.

Here we have to use the observation that filters $\mathcal{F}$ as those in item $(i i)$ above are Hurewicz (being a continuous image of $\mathcal{B} \times \mathcal{P}(\omega)$ ), and the fact that if $\mathcal{B}$ of size $\omega_{1}$ has the Hurewicz property in $V^{\mathbb{P}_{\omega_{2}}}$ then there exists an $\omega_{1}$-club $C \subset \omega_{2}$ such that $\mathcal{B} \in V^{\mathbb{P}_{\alpha}}$ and $\mathcal{B}$ is Hurewicz in $V^{\mathbb{P}_{\alpha}}$ for all $\alpha \in C$.

Now suppose that in $V^{\mathbb{P}_{\omega_{2}}}$ we have a Tychonov space $X$ of size $\omega_{1}$ such that all finite powers of $X$ are Hurewicz. Let $\mathcal{U}$ be an $\omega$-cover of $X . X$ is zero-dimensional because $|X|<2^{\omega}$, and hence passing to a refinement of $\mathcal{U}$, if necessary, we may assume that $\mathcal{U}$ consists of clopen sets. Applying [10, Proposition, p. 156] we can find a countable $\mathcal{V}=\left\{U_{n}: n \in \omega\right\} \subset \mathcal{U}$ which is an $\omega$-cover of $X$. Now consider the map $\psi: X \rightarrow \mathcal{P}(\omega), \psi: x \mapsto$ $\left\{n \in \omega: x \in U_{n}\right\}$. It follows from the above that $\psi$ is continuous and $\psi[X]$ is centered. Since all finite powers of $X$ are Hurewicz, such are also all finite powers of $\psi[X]$, and hence also all finite powers of $\mathcal{B}=\left\{\cap \mathcal{Y}: \mathcal{Y} \in[\psi[X]]^{<\omega}\right\}$ are Hurewicz as well because the latter is a countable union of continuous images of finite powers of $\psi[X]$. Thus the filter $\langle\psi[X]\rangle$ has the Hurewicz base $\mathcal{B}$ of size $|\psi[X]| \leq|X|=\omega_{1}$, and consequently it has a pseudointersection $J \in[\omega]^{\omega}$ by (ii) above. Therefore $J \subset^{*} \psi(x)$ for all $x \in X$, which means that $\left\{U_{n}: n \in J\right\}$ is a $\gamma$-cover of $X$. This completes the proof.

Finally, we shall show that $\mathfrak{p}=\mathfrak{b}<\mathfrak{c}$ holds in any model of Corollary 1.5 (and hence also in those of Theorem 4.3).

Observation 4.4. If $\mathfrak{p}<\mathfrak{b}$, then there exists a $\mathfrak{b}$-scale set which is not a $\gamma$-space.

Proof. It is easy to see that any centered subset $X$ of $[\omega]^{\omega}$ without a pseudointersection is not a $\gamma$-space: consider the open $\omega$-cover $\left\{O_{n}: n \in \omega\right\}$ of $X$, where $O_{n}=\{x: n \in x\}$. Thus there exists $X \subset[\omega]^{\omega}$ of size $\mathfrak{p}$ which is not a $\gamma$-space (this fact has been attributed to [9] in [15]). Now let $\left\{s_{\alpha}: \alpha<\mathfrak{b}\right\}$ be a $\mathfrak{b}$-scale such that 3 divides $s_{\alpha}(n)$ for all $\alpha, n$. Since $s_{\alpha}<^{*} s_{\mathfrak{p}}$ for all $\alpha<\mathfrak{p}$ and $\mathfrak{p}$ is regular, there exists $n$ such that the set $I_{n}=\left\{\alpha: s_{\alpha}(m)<s_{\mathfrak{p}}(m)\right.$ for all $m \geq n$ \} has size $\mathfrak{p}$. Without loss of generality we may assume that $n=0$, otherwise just redefine $s_{\alpha}(k)$ to be equal to $3 k$ for all $\alpha \in I_{n}$ and $k<n$ and 
note that resulting functions still form a $\mathfrak{b}$-scale. Also, we can additionally assume that $I_{0}=\mathfrak{p}$ because otherwise we can set $s_{\alpha}^{\prime}=s_{\xi_{\alpha}}$, where $\xi_{\alpha}$ is the $\alpha$ th element of $I_{0}$, and consider the $\mathfrak{b}$-scale $\left\{s_{\alpha}^{\prime}: \alpha<\mathfrak{p}\right\} \cup\left\{s_{\alpha}: \alpha \geq \mathfrak{p}\right\}$.

Let us write $X$ in the form $\left\{x_{\alpha}: \alpha<\mathfrak{p}\right\}$ and set $t_{\alpha}(n)$ to be the even (resp. odd) element in the set $\left\{s_{\alpha}(n), s_{\alpha}(n)+1\right\}$ if $n \in x_{\alpha}$ (resp. if $n \notin x_{\alpha}$ ). It follows that $t_{\alpha} \leq^{*} s_{\beta}$ for all $\alpha<\mathfrak{p}$ and $\beta \geq \mathfrak{p}$, and $t_{\alpha} \leq^{*} t_{\beta}$ for all $\alpha<\beta<\mathfrak{p}$. Thus $T:=\left\{t_{\alpha}: \alpha<\mathfrak{p}\right\} \cup\left\{s_{\alpha}: \alpha \geq \mathfrak{p}\right\}$ is a $\mathfrak{b}$-scale. Moreover, $T_{0}:=\left\{t_{\alpha}: \alpha<\mathfrak{p}\right\}$ is a closed subset of $T \cup[\omega]<\omega$ because $T_{0}=T \cap K$ for the compact subset $K=\left\{a \in[\omega]^{\omega}: a(n) \leq s_{\mathfrak{p}}(n)\right.$ for all $\left.n\right\}$ of $\mathcal{P}(\omega)$. Since $T_{0}$ can be continuously mapped onto $X$ (using the parity), it is not a $\gamma$-space, and hence $T \cup[\omega]^{<\omega}$ also fails to be a $\gamma$-space because this property is inherited by closed subspaces.

\section{Turning Sets of ReAls into Hurewicz SPACES Without ADDing DOMINATING REALS}

It has been proven in [21] that after adding $\omega_{1}$-many Cohen reals any set of ground model reals becomes Menger. The same argument proves that after iterating with finite supports c.c.c. posets adding dominating reals uncountably many steps, each set of ground model reals becomes Hurewicz. The natural question which arises here is which sets of reals can be made Hurewicz by a forcing not adding dominating reals.

A subset $Y$ of $\omega^{\omega}$ is said to be finitely dominating if the set $\{\operatorname{maxfin}(F): F \in$ $\left.[Y]^{<\omega}\right\}$ is dominating, where $\operatorname{maxfin}(F)$ is the coordinatewise maximum of $F$. It was shown in [23] that a space $X \subset \omega^{\omega}$ has the Scheepers property if and only if any continuous image $Y \subset \omega^{\omega}$ of $X$ is not finitely dominating. It is clear that if a finitely dominating set $Y$ becomes bounded in $V^{\mathbb{P}}$ for some poset $\mathbb{P}$, then $\mathbb{P}$ adds a dominating real. It is a classical result of Hurewicz [15, Theorem 4.3] that $X \subset \omega^{\omega}$ is Hurewicz if and only if all its continuous images $Y \subset \omega^{\omega}$ are bounded. Thus if a non-Scheepers subspace $X$ of $\omega^{\omega}$ becomes Hurewicz in $V^{\mathbb{P}}$ for certain $\mathbb{P}$, then $\mathbb{P}$ adds a dominating real. We do not know whether the converse implication is true.

Question 5.1. Let $X \subset \omega^{\omega}$ be a Scheepers space. Is there a (c.c.c.) poset $\mathbb{P}$ which does not add dominating reals and is such that $X$ is Hurewicz in $V^{\mathbb{P}}$ ?

The following result may be thought of as a step towards answering Question 5.1.

Theorem 5.2. Let $\mathcal{G}$ be a Menger filter. Then there exists a c.c.c. poset $\mathbb{P}_{\mathcal{G}}$ which does not add dominating reals and is such that the filter $\mathcal{G}^{\prime}=\bigcup_{G \in \mathcal{G}} \uparrow G$ generated by $\mathcal{G}$ in $V^{\mathbb{P}_{\mathcal{G}}}$ is Hurewicz in $V^{\mathbb{P}_{\mathcal{G}}}$.

Proof. We shall divide the proof into a sequence of auxiliary statements.

Lemma 5.3. Let $n \in \omega$ and $\mathcal{F}_{i}$ be a filter for all $i \in n$. If $\prod_{i \in n} \mathcal{F}_{i}$ is Menger, then $\mathbb{P}=\prod_{i \in n} \mathbb{M}_{\mathcal{F}_{i}}$ does not add a dominating real.

Proof. The proof will be similar to that of the "if" part of Theorem 1.4. However, we shall present it for the sake of completeness. 
Suppose, to the contrary, that $\dot{g}$ is a $\mathbb{P}$-name for a dominating function. An element of $\mathbb{P}$ may be naturally identified with a sequence $\langle\vec{s}, \vec{F}\rangle$, where $\vec{s}=$ $\langle s(i): i \in n\rangle \in\left([\omega]^{<\omega}\right)^{n}$ and $\vec{F}=\langle F(i): i \in n\rangle \in \prod_{i \in n} \mathcal{F}_{i}$, and $\langle s(i), F(i)\rangle \in$ $\mathbb{M}_{\mathcal{F}_{i}}$ for all $i$.

For every $f \in \omega^{\omega}$ let us find $n_{f} \in \omega$ and a condition $\left\langle\vec{s}^{f}, \vec{F}^{f}\right\rangle$ forcing $f(n)<\dot{g}(n)$ for all $n \geq n_{f}$. Since $\omega^{\omega}$ cannot be covered by a countable family of non-dominating sets, we may assume that $\vec{s}^{f}$ and $n_{f}$ do not depend on $f$, i.e., $\vec{s}^{f}=\vec{s}_{*}$ and $n_{f}=n_{*}$ for all $f \in \omega^{\omega}$.

For every $m \in \omega$ let $\mathcal{S}_{m}$ be the set of those $\vec{s}=\langle s(i): i \in n\rangle \in\left([\omega]^{<\omega}\right)^{n}$ such that $\max s_{*}(i)<\min s(i)$ for all $i$, and there exists $\vec{F}_{\vec{s}}$ such that $\left\langle\left\langle s_{*}(i) \cup\right.\right.$ $\left.s(i): i \in n\rangle, \vec{F}_{\vec{s}}\right\rangle$ forces $\dot{g}(m)$ to be equal to some $g_{\vec{s}}(m)$. It is clear that for every $\vec{F} \in \prod_{i \in n} \mathcal{F}_{i}$ there exists $\vec{s} \in \mathcal{S}_{m}$ such that $s(i) \subset F(i)$ for all $i \in n$. In other words,

$$
\mathcal{U}_{m}:=\left\{\prod_{i \in n} \uparrow s(i):\langle s(i): i \in n\rangle \in \mathcal{S}_{m}\right\}
$$

is an open cover of $\prod_{i \in n} \mathcal{F}_{i}$. Since the latter product is Menger, for every $m$ there exists a finite $\mathcal{V}_{m} \subset \mathcal{U}_{m}$ such that $\prod_{i \in n} \mathcal{F}_{i} \subset \bigcup_{m>l} \bigcup \mathcal{V}_{m}$ for all $l \in \omega$. Let $\mathcal{T}_{m} \in\left[\mathcal{S}_{m}\right]^{<\omega}$ be such that $\mathcal{V}_{m}=\left\{\prod_{i \in n} \uparrow s(i):\langle s(i): i \in n\rangle \in \mathcal{T}_{m}\right\}$ and $f(m)=\max \left\{g_{\vec{s}}(m): \vec{s} \in \mathcal{T}_{m}\right\}$. Let also $m \geq n_{*}$ be such that $\vec{F}^{f} \in$ $\prod_{i \in n} \uparrow s(i)$ for some $\langle s(i): i \in n\rangle \in \mathcal{T}_{m}$. It follows that $s(i) \subset F^{f}(i)$ for all $i \in n$, and hence

$$
p:=\left\langle\left\langle s_{*}(i) \cup s(i): i \in n\right\rangle,\left\langle F^{f}(i) \cap F_{\vec{s}}(i): i \in n\right\rangle\right\rangle
$$

is a condition in $\mathbb{P}$ stronger than both $\left\langle\vec{s}_{*}, \vec{F}^{f}\right\rangle$ and $\left\langle\left\langle s_{*}(i) \cup s(i): i \in n\right\rangle, \vec{F}_{\vec{s}}\right\rangle$. However, $\left\langle\left\langle s_{*}(i) \cup s(i): i \in n\right\rangle, \vec{F}_{\vec{s}}\right\rangle$ forces $\dot{g}(m)=g_{\vec{s}}(m) \leq f(m)$, whereas $\left\langle\vec{s}_{*}, \vec{F}^{f}\right\rangle$ forces $\dot{g}(m)>f(m)$, a contradiction.

Corollary 5.4. Let $\mathscr{F}$ be a collection of filters on $\omega$. If $\prod_{i \in n} \mathcal{F}_{i}$ is Menger for any $n \in \omega$ and $\left\langle\mathcal{F}_{i}: i \in n\right\rangle \in \mathscr{F}^{n}$, then $\mathbb{P}=\prod_{\mathcal{F} \in \mathscr{F}} \mathbb{M}_{\mathcal{F}}$ with finite supports does not add a dominating real.

Proof. Since $\mathbb{P}$ is c.c.c. [14, Theorem 15.15], if $\mathbb{P}$ added a dominating real then there would exist a countable $\mathscr{F}^{\prime} \in\left[\mathscr{F}^{\omega}\right.$ such that $\mathbb{P}^{\prime}=\prod_{\mathcal{F} \in \mathscr{F}} \mathbb{M}_{\mathcal{F}}$ adds a dominating real. The latter product may be viewed as a finite support iteration whose initial segments are equivalent to finite products of Mathias posets with respect to elements of $\mathscr{F}^{\prime}$, and hence these initial segments preserve $V \cap \omega^{\omega}$ unbounded by our assumption. But then by [3, Lemma 6.5.7] we have that $V \cap \omega^{\omega}$ is unbounded in $V^{\mathbb{P}^{\prime}}$ as well, a contradiction.

In general it is a notorious open question whether it is consistent that the Menger property is preserved by finite products. The following simple statement gives the answer in the case of filters.

Claim 5.5. Let $\mathcal{F}$ be a Menger (Hurewicz) filter. Then all finite powers of $\mathcal{F}$ are Menger (Hurewicz). 
Proof. Let us fix $n \in \omega$ and consider the map $\phi: \mathcal{F} \times \mathcal{P}(\omega)^{n} \rightarrow \mathcal{P}(\omega)^{n}$ assigning to $\left\langle F ; A_{0}, \ldots, A_{n-1}\right\rangle$ the sequence $\left\langle F \cup A_{0}, \ldots, F \cup A_{n-1}\right\rangle$. It is clear that the range of $\phi$ is $\mathcal{F}^{n}$. Since the Menger (Hurewicz) property is preserved by products with compact spaces and continuous images, we conclude that $\mathcal{F}^{n}$ is Menger (Hurewicz).

Set $\mathbb{P}_{\mathcal{G}}=\prod_{\alpha \in \omega_{1}} \mathbb{M}_{\mathcal{G}}$ with finite supports and let $\vec{X}=\left\langle X_{\alpha}: \alpha<\omega_{1}\right\rangle$ be the sequence of generic reals added by $\mathbb{P}_{\mathcal{G}}$. By Claim 5.5 and Corollary 5.4 we have that $\mathbb{P}_{\mathcal{G}}$ does not add dominating reals. Let $R=\bigcap_{n \in \omega} O_{n} \in V[\vec{X}]$ be a $G_{\delta}$ subset containing $\mathcal{G}^{\prime}$. By Claim 2.1 we may assume that $O_{n}=\bigcup_{a \in A_{n}} \uparrow a$ for some $A_{n} \in\left(\mathcal{G}^{<\omega}\right)^{+}$. Let $\alpha \in \omega_{1}$ be such that $\left\langle A_{n}: n \in \omega\right\rangle \in V\left[\left\langle X_{\xi}: \xi<\right.\right.$ $\alpha\rangle]$. Since $X_{\alpha}$ is generic over $V\left[\left\langle X_{\xi}: \xi<\alpha\right\rangle\right]$, for every $n \in \omega$ there exist infinitely many $a \in A_{n}$ such that $a \subset X_{\alpha}$. In other words, $\bigcup_{n \in \omega} \uparrow\left(X_{\alpha} \backslash n\right) \subset$ $R$. On the other hand, $\mathcal{G}^{\prime} \subset \bigcup_{n \in \omega} \uparrow\left(X_{\alpha} \backslash n\right)$. Thus we have found a $\sigma$ compact set containing $\mathcal{G}^{\prime}$ and contained in $R$. By [15, Theorem 5.7] this completes our proof.

We do not know whether $\mathcal{G}$ itself becomes Hurewicz in the forcing extension by $\mathbb{P}_{\mathcal{G}}$.

Remark 5.6. Let $\mathscr{F}$ be a family of filters satisfying the premises of Corollary 5.4. The proof of Theorem 5.2 actually allows us fo find a poset $\mathbb{P}$ which does not add dominating reals and such that $\uparrow \mathcal{F}$ is Hurewicz in $V^{\mathbb{P}}$ for all $\mathcal{F} \in \mathscr{F}$.

Acknowledgements. This research was supported by the Slovenian Research Agency grants P1-0292 and J1-5435, the Austrian Academy of Sciences APART Program, the Austrian Science Fund grant I 1209-N25, the GAAVČR grants IAA100190902 and RVO 67985840, and the Czech Ministry of Education grant 7AMB13AT011 Combinatorics and Forcing.

\section{REFERENCES}

[1] Arkhangel'skiur, A., Hurewicz spaces, analytic sets and fan tightness of function spaces, Dokl. Akad. Nauk SSSR 287 (1986), 525-528. (In Russian)

[2] Aubrey, J., Combinatorics for the dominating and unsplitting numbers, J. Symbolic Logic Volume 69, Issue 2 (2004), 482-498.

[3] Bartoszyński, T.; Judah, H., Set Theory: On the structure of the real line, A. K. Peters, Massachusetts: 1995.

[4] Bartoszyński, T.; Tsaban, B., Hereditary topological diagonalizations and the Menger-Hurewicz conjectures, Proc. Amer. Math. Soc. 134 (2006), 605-615.

[5] Blass, A., Near Coherence of filters, I. Cofinal equivalence of models of arithmetic, Notre Dame J. Formal Logic 27 (1986), 579-591.

[6] Brendle, J., Mob families and mad families, Arch. Math. Logic 37 (1998), 183-197.

[7] Canjar, R., Mathias forcing which does not add dominating reals, Proc. Amer. Math. Soc. 104 (1988), 1239-1248.

[8] Canjar, R., Cofinalities of countable ultraproducts: the existence theorem, Notre Dame J. Formal Logic 30 (1989), 539-542.

[9] Galvin, F.; Miller, A.W., $\gamma$-sets and other singular sets of real numbers, Topology Appl. 17 (1984), 145-155.

[10] Gerlits, J., Nagy, Zs., Some properties of $C(X)$, I, Topology Appl. 14 (1982), 151161.

[11] Guzmán, O.; Hrušák, M.; Martínez, A.A., Canjar filters, Notre Dame J. Formal Logic, to appear. 
[12] Hrušák, M.; Minami, H., Laver-Prikry and Mathias-Prikry type forcings, Ann. Pure Appl. Logic 165 (2014), 880-894.

[13] Hurewicz, W., Über eine Verallgemeinerung des Borelschen Theorems, Math. Z. 24 (1925), 401-421.

[14] Jech, T., Set theory. The third millennium edition, revised and expanded. Springer Monographs in Mathematics. Springer-Verlag, Berlin, 2003.

[15] Just, W.; Miller, A.W.; Scheepers, M.; Szeptycki, P.J., The combinatorics of open covers II, Topology Appl. 73 (1996), 241-266.

[16] Laflamme, C., Forcing with filters and complete combinatorics, Ann. Pure Appl. Logic 42 (1989), 125-163.

[17] Laflamme, C.; Leary, C.C., Filter games on $\omega$ and the dual ideal, Fund. Math. 173 (2002), 159-173.

[18] Orenshtein, T.; Tsaban, B., Linear $\sigma$-additivity and some applications, Trans. Amer. Math. Soc. 363 (2011), 3621-3637.

[19] Repovš, D.; Tsaban, B.; Zdomskyy, L., Hurewicz sets of reals without perfect subsets, Proc. Amer. Math. Soc. 136 (2008), 2515-2520.

[20] Scheepers, M., Combinatorics of open covers. I. Ramsey theory, Topology Appl. 69 (1996), 31-62.

[21] Scheepers, M.; Tall, F.D., Lindelöf indestructibility, topological games and selection principles, Fund. Math. 210 (2010), 1-46.

[22] Shelah, S., On cardinal invariants of the continuum, Axiomatic set theory (Boulder, Colo., 1983) 31 (1984), 183-207

[23] Tsaban, B., A diagonalization property between Hurewicz and Menger, Real Anal. Exchange 27 (2001/2002), 757-763.

[24] Tsaban, B.; Zdomskyy, L., Scales, fields, and a problem of Hurewicz, J. Eur. Math. Soc. 10 (2008), no. 3, 837-866.

[25] Vaughan, J., Small uncountable cardinals and topology, in: Open problems in topology (J. van Mill, G.M. Reed, Eds.), Elsevier Sci. Publ., Amsterdam 1990, pp. 195-218.

Institute of Mathematics of the Academy of Sciences of the Czech Republic, Žitná 25, Praha 1, Czech Republic

E-mail address: david.chodounsky@matfyz.cz

Faculty of Education, and Faculty of Mathematics and Physics, University of Ljubljana \& Institute of Mathematics, Physics and Mechanics, Luubljana, SLOVENIA 1001.

E-mail address: dusan.repovs@guest.arnes.si

Kurt Gödel Research Center for Mathematical Logic, University of Vienna, Währinger Strasse 25, A-1090 Wien, Austria.

E-mail address: lzdomsky@gmail.com 